\title{
Critical factors for liposome-incorporated tumour-associated antigens to induce protective tumour immunity to SL2 lymphoma cells in mice
}

\author{
Joep J. Bergers ${ }^{*}$, Willem Den Otter ${ }^{2}$, Hub F. J. Dullens ${ }^{2}$, Jan Willem De Groot ${ }^{2}$, Peter A. Steerenberg ${ }^{3}$, \\ Monique W. H. Mimpen', Daan J. A. Crommelin1 \\ ${ }^{1}$ Department of Pharmaceutics, Faculty of Pharmacy, University of Utrecht, P. O. Box 80.082, 3508 TB Utrecht, The Netherlands \\ ${ }^{2}$ Department of Pathology, University Hospital Utrecht, P. O. Box 85.500, 3508 GA Utrecht, The Netherlands \\ ${ }^{3}$ Laboratory for Pathology, National Institute of Public Health and Environmental Protection, P. O. Box 1, 3720 BA Bilthoven, The Netherlands
}

Received: 4 January 1993/Accepted: 3 March 1993

\begin{abstract}
Physical and immunogenic properties of reconstituted membranes designed for the presentation of tumour-associated antigens (TAA) to the immune system are described. Proteins and lipids of crude membranes of SL2 murine lymphosarcoma cells were partially solubilized with octylglucoside. Reconstituted membranes, consisting mainly of unilamellar vesicles with a diameter of $0.03-0.15 \mu \mathrm{m}$, were formed by detergent removal and were purified by floatation in a discontinuous sucrose gradient to remove non-lipid-bound protein. Subcutaneous immunization of syngeneic mice with reconstituted membranes or with purified reconstituted membranes induced protection against an intraperitoneal challenge with $10^{3}$ viable SL2 cells. Reconstituted membranes were more immunogenic than crude membranes in immunoprotection experiments when compared on the basis of protein dose. Detergent removal was required to obtain an immunogenic presentation form of SL2 membrane antigens and to avoid toxicity associated with the detergent. Reconstitution of SL2 membranes in the presence of exogenous phospholipid slightly increased the fraction of protein that associated with the reconstituted membranes. However, the immunogenicity of the solubilized membrane TAA was not significantly affected by the presence of exogenous phospholipid. The reconstitution procedure described may be useful in identifying membrane factors required for the induction of immune responses against TAA. The versatility of the system may be employed to develop safe alternatives for whole-cell vaccines.
\end{abstract}

Key words: Tumour vaccine - Liposomes - Reconstituted membranes - Lymphoma cells

\footnotetext{
* Present address: Department of Clinical Microbiology, Erasmus University, P. O. Box 1738, 3000 DR Rotterdam, The Netherlands

Correspondence to: J. J. Bergers, at present address
}

\section{Introduction}

Liposomes, membrane-like vesicles containing one or more phospholipid (PL) bilayers surrounding aqueous compartments, have generated considerable interest as vehicles for the presentation of antigens to the immune system (reviewed by Bergers et al. [4], Gregoriadis [15] and Kersten et al. [23]). As the liposomal characteristics, such as charge, bilayer fluidity, particle size and antigen density, can be varied, liposomes provide valuable tools to investigate mechanisms involved in antigen recognition and induction of immunity. Such properties may be employed to prepare tailor-made vaccines for human and veterinary use [15].

Immune reactions against chemically induced murine sarcomas were demonstrated more than three decades ago [32]. Antibodies against the autologous tumour in the serum of cancer patients [29] and tumour-infiltrating lymphocytes in or around the tumour [2] indicate that at least some human tumours are recognized by the immune system. However, it has been extremely difficult to exploit the immune system for developing strategies for cancer treatment. One approach is active specific immunotherapy with vaccines containing tumour-associated antigens (TAA). The results of clinical studies with tumour vaccines composed of a combination of adjuvants with irradiated tumour cells, tumour cell lysates, or extracted TAA of tumour cells (TAA extracts) suggest that these vaccines can slow down the progression of some cancers and can increase the survival time of patients $[7,19,27,36]$.

The TAA responsible for inducing tumour rejection are (glyco)proteins or glycolipids expressed on the cell surface. Crude TAA extracts, prepared by treatment of tumour cells with low butanol concentrations or hypertonic $\mathrm{KCl}$ solutions, for example, can induce protective tumour immunity in murine models [26]. Despite numerous efforts, only a limited number of such TAA have been characterized successfully [35]. The potential of TAA extracts to induce immunity is generally decreased compared to irradiated tumour cells or purified plasma membranes. It is, therefore, of interest to investigate the immunogenicity of 
cell-membrane antigens presented in a fashion more similar to that of intact cells, that is in a more membrane-like structure. When TAA are solubilized by a detergent that leaves the hydrophobic part of proteins intact, insertion of TAA into a liposome structure may offer possibilities for presentation of the antigenic structures.

As shown in a previous paper [3], protective tumour immunity against the weakly immunogenic, spontaneously arising SL2 lymphosarcoma can be induced by immunization of syngeneic mice with reconstituted membranes. Reconstituted membranes consist of liposome-like structures prepared from cell membrane constituents. They are formed by removal of the detergent from solubilized membrane lipids and proteins. However, it was observed that, depending on the detergent used, only $50 \%-80 \%$ of the solubilized proteins were associated with the reconstituted membranes. This made it impossible to define the exact role of the membrane-associated proteins in the induction of protection, as only some of the proteins in the vaccine were membrane-associated.

In this study, free, non-membrane-associated proteins present in the reconstituted membranes were separated from membrane-associated proteins (purified reconstituted membranes), and both fractions were tested for their potential to induce tumour immunity. The immunogenicity of the purified reconstituted membranes was compared to that of solubilized membrane antigens. As the protein to PL ratio may affect the reconstitution efficiency and the immunogenicity of membrane-presented antigens $[10,38]$, the effect of the addition of exogenous PL to the reconstituted membranes on their immunogenic potential was studied. Considerable attention was paid to the physical and chemical characteristics of the different antigen dispersions used.

\section{Materials and methods}

Mice. Inbred DBA/2 mice were obtained from IFFA-Credo, France. Animals were maintained according to standard conditions in the central. animal facilities of Utrecht University. Male mice were used at an age of $2-3$ months.

Tumours. The DBA/2-derived SL2 lymphosarcoma, which arose spontaneously as an ascitic tumour, was used. The tumour was maintained in syngeneic DBA/2 mice by weekly intraperitoneal (i.p.) passage of $5 \times 10^{5}$ cells. The tumour grows i.p. as an ascitic tumour and subcutaneously (s.c.) as a solid tumour; about $50 \%$ of the animals die from tumour growth after i.p. injection of 1-10 SL2 cells [11].

Preparation of reconstituted membranes. Freshly harvested SL2 cells were obtained 7-8 days after transplantation and were washed three times with RPMI-1640 medium (Flow Laboratories, McLean, Va.). Crude membranes were isolated from these cells as described before [3]. Briefly, tumour cells were disrupted by a freezing/thawing cycle in a hypotonic buffer. Cellular debris and nuclei were removed by low-speed centrifugation and crude membranes were pelleted by high-speed centrifugation. Crude membranes were stored in small aliquots at $-70^{\circ} \mathrm{C}$ until use.

After thawing, crude membranes were partially solubilized by incubation with $150 \mathrm{mM}$ octylglucoside (OG) (Sigma Chemical Co., St. Louis, Mo.) in $10 \mathrm{mM}$ TRIS/ $\mathrm{HCl}, 140 \mathrm{mM} \mathrm{NaCl}, \mathrm{pH} 7.3$, at a protein concentration of $2.0 \mathrm{mg} / \mathrm{ml}$. This detergent is one of the few detergents that combines two characteristics that are very useful for reconstitution experiments: a non-ionic nature and a high critical micelle concentration (the detergent concentration at and above which micelles form). Nonionic detergents generally do not have deleterious effects on the biological activity of proteins. A high critical micelle concentration makes the detergent readily dialysable [17]. After incubation for 30 min with $O G$ with stirring, non-solubilized material was removed by centrifugation at $105000 \mathrm{~g}$ for $45 \mathrm{~min}$. Reconstituted membranes were formed by dialysing the supernatant for $48-72 \mathrm{~h}$ against at least 100 -fold buffer volume, with two changes. All these steps were performed on ice or at $4^{\circ} \mathrm{C}$. For each immunization the reconstituted membranes were freshly prepared.

Purification of reconstituted membranes. Reconstituted membranes of SL2 cells were purified by floatation in a discontinuous sucrose gradient. Solid sucrose was added to $3.0 \mathrm{ml}$ membrane suspension in a centrifuge tube to a final concentration of $45 \%(\mathrm{w} / \mathrm{w})$. This suspension was overlayed with $5.0 \mathrm{ml} \mathrm{35 \%}(\mathrm{w} / \mathrm{w})$ sucrose in TRIS-buffered saline, and then with $1.5 \mathrm{ml}$ TRIS-buffered saline, and was centrifuged for $16 \mathrm{~h}$ at $130000 \mathrm{~g}$. Fractions of $1 \mathrm{ml}$ were collected, starting at the top of the gradient.

To locate membrane-associated proteins in the sucrose gradient, membrane proteins devoid of lipids and lipids devoid of membrane proteins were isolated from crude membranes of SL2 cells and were run in the gradient.

To obtain membrane proteins devoid of lipids, most of the proteins and PL of crude membranes were solubilized by treatment with $2 \%$ deoxycholate (Sigma Chemical Co., St. Louis, Mo.) in $10 \mathrm{mM}$ TRIS/ $\mathrm{HCl}, 140 \mathrm{mM} \mathrm{NaCl}, \mathrm{pH}$ 8.2. The solubilized membranes were passed through a Sephadex G-50 column (Pharmacia, Uppsala, Sweden) equilibrated with buffered $0.2 \%$ deoxycholate $(\mathrm{pH} 8.2$ ) and fractionated. The protein content of each fraction was determined by measuring the absorbance at $280 \mathrm{~nm}$, and the PL content was assessed as described below. Those protein fractions in which no PL could be detected (containing more than $90 \%$ of the total solubilized proteins) were pooled, concentrated by ultrafiltration, and dialysed against TRIS-buffered saline to remove deoxycholate. Subsequently, these proteins were solubilized in $O G$ and subjected to the reconstitution and purification procedures as described for reconstituted membranes.

Lipids devoid of proteins were extracted from crude membranes with chloroform/methanol as described before [3]. Lipids were solubilized in $150 \mathrm{mM} \mathrm{OG}$ and subjected to the reconstitution and purification procedures as described for reconstituted membranes.

For immunoprotection studies, fractions $1-3$ (referred to as the purified reconstituted membranes) and fractions 6-11 (referred to as the remaining fraction) of the gradient were pooled and were dialysed overnight against TRIS-buffered saline to remove the sucrose. Subsequently, the fractions were concentrated by overlaying the dialysis tubes with solid polyethyleneglycol 40,000 (Serva, Heidelberg, Germany). The whole procedure was performed on ice or at $4^{\circ} \mathrm{C}$.

Trypsin treatment of solubilized membranes. Solubilized membranes at a concentration of approximately $0.8 \mathrm{mg}$ protein $/ \mathrm{ml}$ were incubated with $10^{3} \mathrm{U} / \mathrm{ml}$ bovine pancreas trypsin (Sigma Chemical Co., St. Louis, Mo.) for $2 \mathrm{~h}$ at $37^{\circ} \mathrm{C}$. Reconstituted membranes were obtained by removal of the detergent and purified as described above.

Preparation of reconstituted membranes with additional exogenous phospholipid. Purified reconstituted membranes containing additional exogenous PL were prepared as follows. A solution of egg $\mathrm{L}-\alpha-$ phosphatidylcholine type V-E (PtdCho) (Sigma Chemical Co., St. Louis, Mo.) and egg phosphatidylglycerol (PtdGro) (a gift from Nattermann $\mathrm{GmbH}$, Cologne, Germany) (9:1 ratio) in a chloroform/methanol mixture $(9: 1)$ was evaporated to dryness. The lipid film was solubilized in $150 \mathrm{mM}$ OG. A sample containing 3,10 or $30 \mu \mathrm{mol}$ PL was added $/ \mathrm{ml}$ solubilized membranes, which contained approximately $1.0 \mu \mathrm{mol}$ PL extracted from the crude membranes. Purified reconstituted membranes were obtained as described above.

Electron microscopy. Electron microscopy of reconstituted membranes was performed by Dr. J. Röding at the laboratories of Nattermann Phos- 


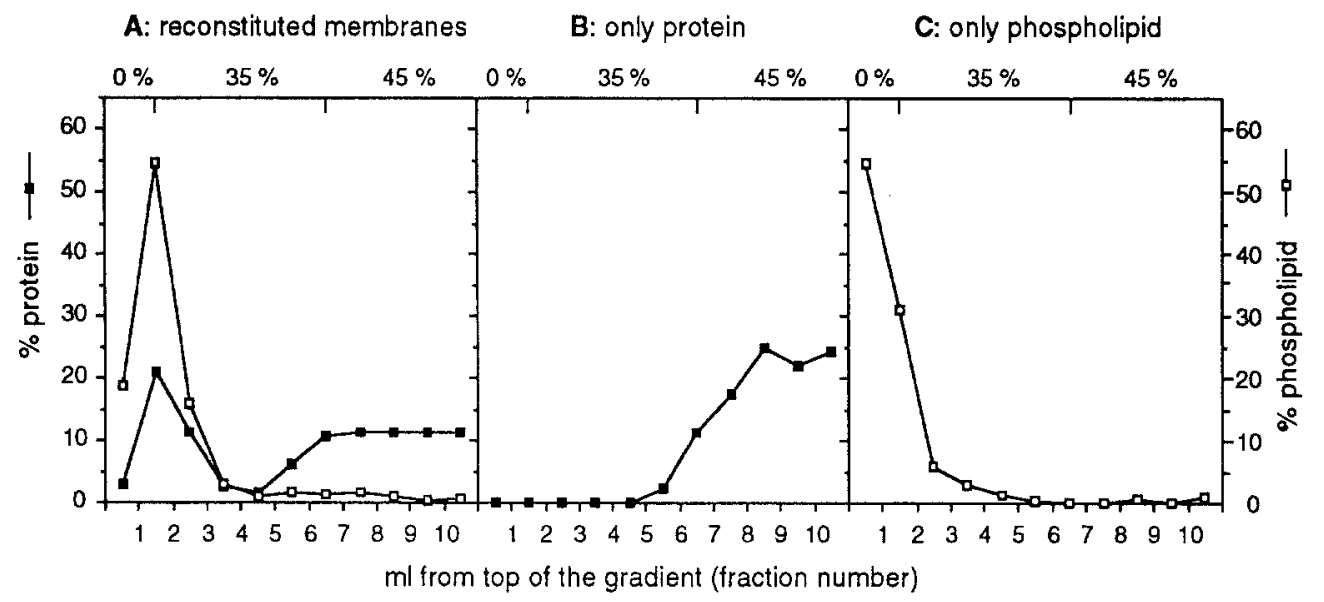

Fig. 1 A - C. Floatation of reconstituted membranes in a discontinuous sucrose gradient. A Crude membranes were solubilized with octylglucoside and reconstituted membranes were obtained by detergent removal. Reconstituted membranes were centrifuged in a discontinuous sucrose gradient for $16 \mathrm{~h}$. The composition of the gradient is depicted above the graph. Fractions of $1 \mathrm{ml}$ were collected from the top of the gradient and analysed for protein ( $\mathbf{a}$ ) and phospholipid $(\square)$ content. B Membrane proteins were separated from lipids by gel filtration of solubilized membrane proteins and were subsequently subjected to the reconstitution and floatation procedure as described for A. C Lipids were obtained from SL2 cells by repeated chloroform/methanol extraction and were subjected to the reconstitution and floatation procedure as described for $\mathbf{A}$ pholipid GmbH, Cologne, Germany. Micrographs were obtained after cryofixation of the dispersions on the grid.

Analytical assays. The protein content was assessed by the method of Wessel and Flügge [39] using bovine serum albumin type V (Sigma Chemical Co., St. Louis, Mo.) as a standard. Lipid phosphorus was determined by the colorimetric method of Fiske and Subbarow [14] after lipid extraction with chloroform/methanol according to the method of Bligh and Dyer [5]. Mean particle size and polydispersity were measured by dynamic light scattering with a Malvern 4700 system using a $25-\mathrm{mW}$ helium/neon laser and the automeasure version 3.2 software (Malvern Ltd., Malvern, UK). The distribution of particle sizes was quantified using the polydispersity index. This index ranges from 0.0 for an entirely monodisperse preparation up to 1.0 for a completely polydisperse preparation. Sodium dodecylsulphate/polyacrylamide gel electrophoresis (SDS-PAGE) was performed according to Laemmli [24] with some modifications described by Jiskoot et al. [22]. Protein was visualized by Coomassie brilliant blue staining.

Immunization. DBA/2 mice were immunized by two s.c. injections with presumptive antigen in a total volume of $0.20 \mathrm{ml}$ on the chest on days $-30,-20$ and -10 . On day 0 , mice were challenged i. p. with $10^{3}$ viable SL2 cells. Survival of mice was monitored daily. The cellular equivalent, that is the amount of protein that can be extracted from a fixed number of tumour cells, was assessed from initial experiments.

Statistical analysis. Differences in survival times of groups of mice were analysed by the non-parametric Wilcoxon rank-sum test. Student's $t$-test was used to test the significance of differences in the percentage protein associated with the reconstituted membranes.

\section{Results}

\section{Characteristics and purification of reconstituted membranes}

Crude cell membranes of SL2 cells were solubilized with OG and non-solubilized material was removed by centrifugation. In a typical experiment, OG solubilized about $40 \%$ of the membrane proteins and about $80 \%$ of the phos-

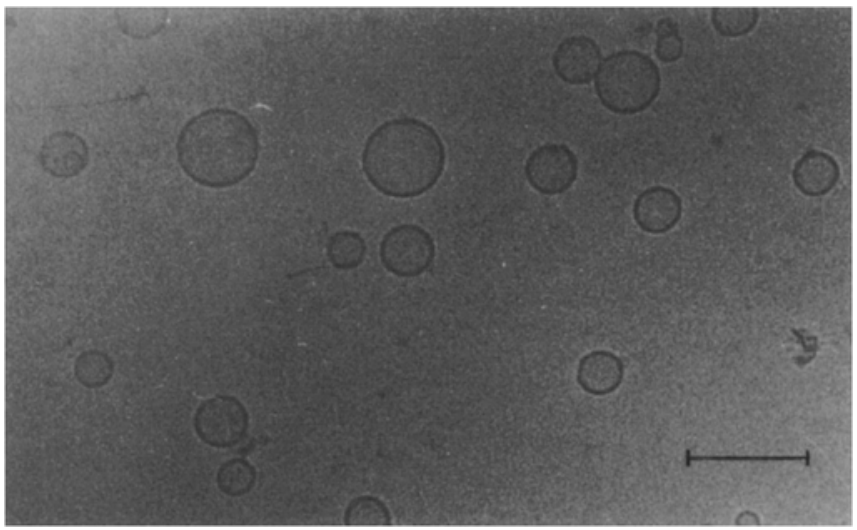

Fig. 2. Electron micrograph (cryofixation) of SL2 reconstituted membranes. Scale bar represents $0.2 \mu \mathrm{m}$

pholipids. Proteins and lipids were allowed to reassociate into membranes by removal of the detergent. Initial characterization of these reconstituted membranes has been described before. Proteins solubilized by OG were separated by SDS-PAGE, and patterns in different runs were compared with those given by the crude membrane proteins. At least 35 different protein bands could be distinguished in the membrane samples. Both the solubilized membranes and the reconstituted membranes exhibited about 18 protein bands; these were also present in the crude membranes. Especially proteins with a low electrophoretic mobility were not solubilized by OG. Storage of membranes or reconstituted membranes at $-20^{\circ} \mathrm{C}$ for 3 months did not produce alterations in the mobility pattern.

Reconstituted membranes were purified by floatation in a discontinuous sucrose gradient to remove proteins that were not associated with the membranes. A typical elution profile of proteins and PL is shown in Fig. 1 A. Approximately $30 \%$ of the proteins and $90 \%$ of the PL were recovered in the upper three fractions. When reconstitution was 
Table 1. Characteristics of reconstituted membranes and purified reconstituted membranes used for the immunization studies

Ratio protein/PL reconstituted membranes $(\mathrm{mg} / \mu \mathrm{mol})^{\mathrm{a}}$

$0.8 \pm 0.1^{b} \quad(n=10)$

Ratio protein/PL purified reconstituted membranes $(\mathrm{mg} / \mu \mathrm{mol})^{\mathrm{c}}$

Protein recovered in the purified reconstituted membranes $(\%)^{\mathrm{d}}$

Protein recovered in the remaining fraction $(\%)^{\mathrm{d}}$

Mean particle size of reconstituted membranes $(\mu \mathrm{m})^{\mathrm{e}}$

Polydispersity index of reconstituted membranese, f

$$
\begin{array}{cc}
0.34 \pm 0.05 & (n=9) \\
31 \pm 5 & (n=21) \\
62 \pm 11 & (n=21)
\end{array}
$$$$
0.14 \pm 0.02 \quad(n=19)
$$$$
0.2-0.3
$$

Mean particle size of purified reconstituted membranes $(\mu \mathrm{m})^{\mathrm{e}}$

$$
0.16 \pm 0.02 \quad(n=16)
$$

Polydispersity index of purified

reconstituted membranes $s^{e, f}$

$$
0.3-0.4
$$

Protein in remaining fraction that could not be pelleted $(\%) \mathrm{g}$

$72 \pm 8 \quad(n=5)$

a PL, phospholipid; SL2 membranes (protein/PL ratio of $1.6 \pm 0.1 \mathrm{mg} / \mu \mathrm{mol}$ ) were solubilized with octylglucoside and reconstituted membranes were obtained by detergent removal

b Mean \pm standard deviation

c Reconstituted membranes were centrifuged in a discontinuous sucrose gradient. The amount of protein and phospholipid were measured in the pooled upper three fractions

d Expressed as the amount of protein measured in this fraction compared to the amount of protein applied to the gradient. Protein recovery was $99 \pm 11 \%$

c Determined with dynamic light scattering

f This index ranges from 0.0 to $1.0 ; 0.0$ denotes a monodisperse and 1.0 an extremely polydisperse system

$\mathrm{g}$ The remaining fraction was centrifuged at $250000 \mathrm{~g}$ for $2 \mathrm{~h}$ and the amount of protein in the supernatant was determined

performed with only proteins and this material was run in a sucrose gradient, $100 \%$ of the proteins remained at the bottom of the gradient (Fig. 1B). Centrifugation of reconstituted membranes consisting mainly of SL2 membrane lipids in a sucrose gradient resulted in floatation of all the PL (Fig. 1C). This was also observed for empty liposomes composed of PtdCho/PtdGro (9:1) (data not shown).

Therefore, the proteins in the upper three fractions were considered to be associated with the reconstituted membranes prepared from the cell membrane lipids. After recentrifugation of the pooled three upper fractions in a discontinuous sucrose gradient, the proteins were quantitatively recovered in these fractions (data not shown). This implies that the proteins are stably associated with the reconstituted membranes and that these fractions are not contaminated with proteins that are not associated with the reconstituted membranes.

An electron micrograph (made after cryofixation on the grid) of the reconstituted membranes obtained from the pooled upper three fractions is shown in Fig. 2. The reconstituted membranes consisted of liposomal structures with a spherical shape and with diameters ranging from
A: (purified) reconstituted membranes (fractions 1-3)

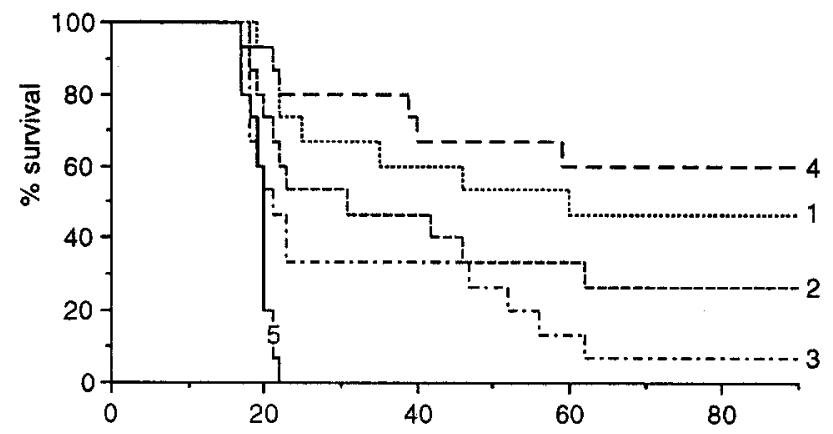

B: remaining fraction (fractions 6-11)

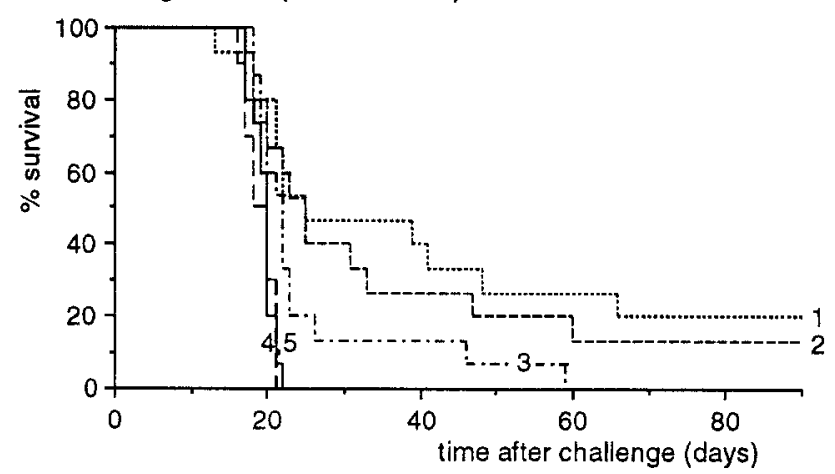

Fig. 3 A, B. Induction of protective tumour immunity by immunization with purified reconstituted membranes. Reconstituted membranes prepared from SL2 membranes were centrifuged in a discontinuous sucrose gradient. The reconstituted membranes (pooled fractions $1-3$; see Fig. 1 A) and the remaining fraction (pooled fractions 6-11; see Fig. 1 A) were collected, dialysed, and concentrated. Furthermore, the remaining fraction was centrifuged at $250000 \mathrm{~g}$ for $2 \mathrm{~h}$ to spin down any particulate material present. Mice were immunized three times s. c. $(10-$ day intervals). Ten days after the last immunization the animals were challenged i.p. with $10^{3}$ SL2 cells. Protein dose (cellular equivalents)/immunization: A $40 \mu \mathrm{g}\left(2.5 \times 10^{7}\right)$ purified reconstituted membranes $(1, \cdots) ; 16 \mu \mathrm{g}\left(1.0 \times 10^{7}\right)$ purified reconstituted membranes $(2$, $---) ; 4 \mu \mathrm{g}\left(2.5 \times 10^{6}\right)$ purified reconstituted membranes $(3,-\cdot-)$ $125 \mu \mathrm{g}\left(2.5 \times 10^{7}\right)$ reconstituted membranes $(4,--)$; no immunization $(5,-)$; B $75 \mu \mathrm{g}\left(2.5 \times 10^{7}\right)$ remaining fraction $(1, \cdots) ; 30 \mu \mathrm{g}\left(1.0 \times 10^{7}\right)$ remaining fraction $(2, \ldots--) ; 8 \mu \mathrm{g}\left(2.5 \times 10^{6}\right)$ remaining fraction (3, $-.-) ; 50 \mu \mathrm{g}\left(2.5 \times 10^{7}\right)$ supernatant after pelleting particulate material of the remaining fraction $(4,--)$; no immunization $(5,-)$. 10-15 mice/group

$0.03 \mu \mathrm{m}$ to $0.15 \mu \mathrm{m}$. About $95 \%$ of the vesicles were unilamellar.

The physical nature of the remaining (bottom) fractions (fractions 6-11; fractions 4 and 5 were discarded) is less clear. About $10 \%$ of the PL of the reconstituted membranes applied to the sucrose gradient was found in these fractions. After removal of sucrose, $72 \pm 8 \%(n=5)$ of the proteins present in these fractions could not be pelleted by centrifugation at $250000 \mathrm{~g}$ for $2 \mathrm{~h}$. No PL could be detected in the supernatant. This implies that, apart from "soluble" proteins, protein-containing particles with a relatively high density, probably protein-(lipid) aggregates and/or liposomes with a relatively high protein content and, consequently, a relatively high density $[9,12]$, are present in the bottom fractions. The characteristics of reconstituted membranes used for the in vivo experiments are summarized in Table 1. 


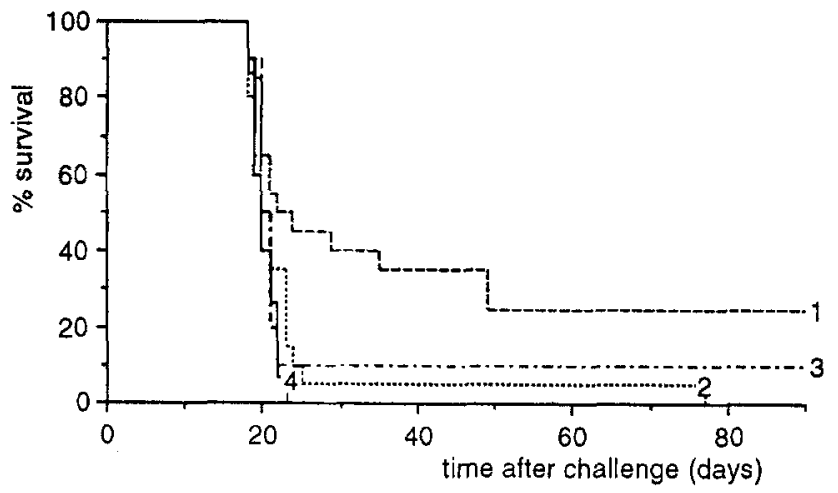

Fig. 4. Effect of the presentation form on the immunogenicity of SL2 membrane antigens. Reconstituted membranes prepared from SL2 membranes were purified by centrifugation in a discontinuous sucrose gradient. The immunization schedule was the same as that described in Fig. 3. Protein dose/immunization was $16 \mu \mathrm{g}$. Purified reconstituted membranes $(1, \ldots--)$; purified reconstituted membranes solubilized in $40 \mathrm{mM}$ octylglucoside $(2, \cdots) ; 40 \mathrm{mM}$ octylglucoside only $(3,-\cdot-)$; no immunization $(4,-)$. 10-20 mice/group

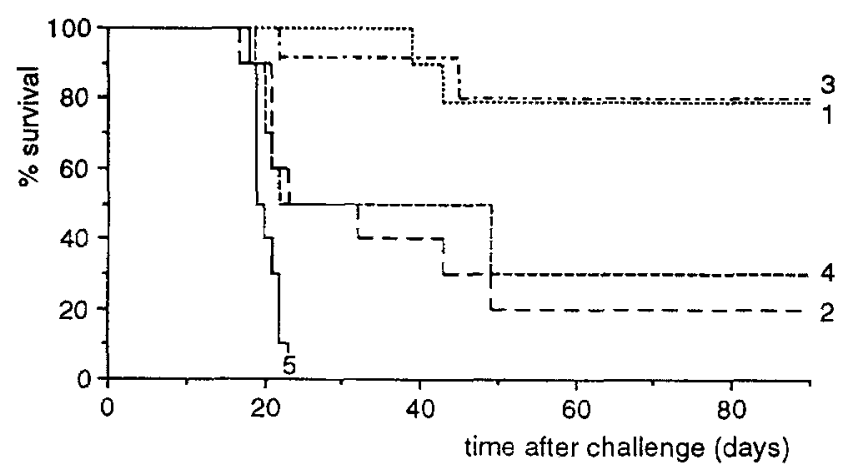

Fig. 5. Comparison of the immunoprotective effects of various preparations of SL2 cells on the basis of protein. For the preparation method and immunization schedule see Fig. 3. Protein dose/immunization was $16 \mu \mathrm{g} .1 .5 \times 10^{5} \mathrm{SL} 2$ cells irradiated with 50 Gy $\gamma$ irradiation $(1, \cdots)$; crude membranes $(2,--)$; reconstituted membranes $(3,-\cdot)$; purified reconstituted membranes $(4,---)$; no immunization $(5,-), 10$ mice/group

\section{Protective tumour immunity induced by purified reconstituted membranes}

The potential to induce protection against a challenge with $10^{3}$ SL2 cells was determined for (a) reconstituted membranes, (b) purified reconstituted membranes (the pooled fractions 1-3 shown in Fig. 1 A), (c) the remaining fraction (the pooled fractions 6-11 shown in Fig. 1 A), and (d) the supernatant after particulate material present in the remaining fraction had been pelleted. DBA $/ 2$ mice were immunized three times s.c. (10-day intervals). Ten days after the last immunization, mice were challenged i. p. with $10^{3}$ syngeneic viable tumour cells. The results, depicted in Fig. 3, show that all non-immunized mice died within 22 days. Mice immunized with $2.5 \times 10^{7}$ cellular equivalents of reconstituted membranes $(125 \mu \mathrm{g}$ protein), purified reconstituted membranes ( $40 \mu \mathrm{g}$ protein), or the remaining fraction ( $75 \mu \mathrm{g}$ protein) survived significantly
Table 2. Effect of trypsin treatment on the protective tumour immunity induced by purified reconstituted membranes

\begin{tabular}{|c|c|c|}
\hline $\begin{array}{l}\text { Type of preparation used for } \\
\text { immunization }^{\mathrm{a}}\end{array}$ & mice & $\begin{array}{l}\mathrm{MST} \pm \mathrm{SD}^{\mathrm{c}} \\
\text { (days) }\end{array}$ \\
\hline $\begin{array}{l}\text { stituted membranes } \\
\text { astituted membranes treated } \\
\text { ne }^{e}\end{array}$ & $\begin{array}{l}0 / 15 \\
0 / 10\end{array}$ & \\
\hline $\begin{array}{l}\text { a Immunization schedule was the sam } \\
\text { b Number of animals alive at day } 40 / \mathrm{t} \\
\text { c Mean survival time } \pm \text { standard devi } \\
\text { d Dose was } 40 \mu \mathrm{g} \text { protein } / \text { immunizat } \\
\text { constituted membranes } \\
\text { e Solubilized membranes were treated } \\
\text { the detergent was removed. The recon } \\
\text { fuged in a discontinuous sucrose grad } \\
\text { constituted membranes were removed, }\end{array}$ & $\begin{array}{l}\text { trypsin at } 3 \\
\text { ted membra } \\
\text { The fraction }\end{array}$ & $\begin{array}{l}\text { for } 2 \mathrm{~h} \text { and } \\
\text { vere centri- } \\
\text { taining re- }\end{array}$ \\
\hline
\end{tabular}

longer than non-immunized mice $(P<0.01$ for all three comparisons). Between $20 \%$ and $60 \%$ of the animals survived for more than 90 days. These animals had no visible tumour and were considered cured. Immunization of mice with purified reconstituted membranes tended to be better than immunization with the remaining fraction, but the differences in survival were not statistically significant at any of the three dose levels tested $(P>0.05$, compared on the basis of cellular equivalents). Mice immunized with the remaining fraction devoid of particulate material could not reject the challenge with SL2 cells; all these animals died within 21 days after tumour challenge. This demonstrates that the protection induced by the remaining fraction is not induced by soluble proteins or small protein micelles, but by particulate material with a relatively high density.

\section{Effect of the presentation vehicle of membrane antigens on the induction of protective tumour immunity}

The effect of the presentation form of SL2 TAA (inserted in a membrane structure or presented as a detergent-protein complex) on the induction of tumour immunity was studied (Fig. 4). Therefore, purified reconstituted membranes were solubilized in $40 \mathrm{mM} \mathrm{OG}$ at a final protein concentration of $80 \mu \mathrm{g} / \mathrm{ml}$. The protection elicited by immunization with these solubilized membranes was significantly decreased with regard to the intact, purified, reconstituted membranes $(P<0.05)$. No significant differences in survival were found between groups of mice treated with the solubilized reconstituted membranes and treated with the solubilizing agent (OG) only. Subcutaneous administration of the preparations containing OG caused inflammatory reactions at the injection sites, which were observed macroscopically and confirmed by microscopical examination. Such reactions were not seen after injections with reconstituted membranes. Usually all non-immunized mice died within 25 days after i.p. injection of $10^{3}$ SL2 cells. Nonspecific increase in the resistance through the administra- 


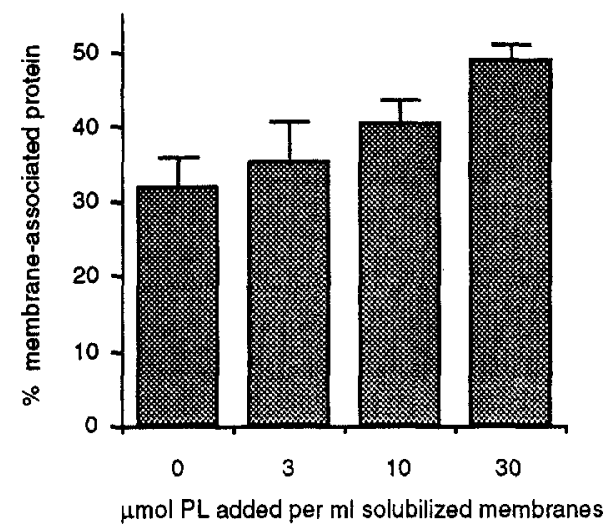

Fig. 6. Effect of the addition of exogenous phospholipid $(P L)$ on the reconstitution efficiency of SL2 membrane proteins. Crude SL2 membranes were solubilized with octylglucoside and the indicated amount of phospholipid (phosphatidylcholine/phosphatidylglycerol at a ratio of 9:1) was added. After detergent removal, the reconstituted membranes were centrifuged in a discontinuous sucrose gradient. The percentage protein associated with the reconstituted membranes was calculated from the amount of protein in the upper three fractions of the gradient devided by the amount of protein applied. Error bars indicate standard deviations (five experiments)

tion of OG may account for the animal that rejected the tumour in the group treated with OG only and, consequently, for the animal that died at day 77 in the group treated with membranes solubilized in OG.

\section{Protective tumour immunity induced by different preparations of SL2 cells}

Results of experiments in which the immunogenicities of irradiated tumour cells, crude membranes, reconstituted membranes, and purified reconstituted membranes were compared on the basis of equal protein doses $(16 \mu \mathrm{g} / \mathrm{immu}-$ nization) are shown in Fig. 5. Immunization of mice with $1.5 \times 10^{5}$ irradiated SL2 cells induced protection against a challenge with $10^{3} \mathrm{SL} 2$ cells. The immunoprotective activity of equal doses of crude membrane proteins was significantly less $(P<0.01)$. However, immunization with reconstituted membranes was significantly more effective than immunization with crude membranes $(P<0.05)$. The protection induced by reconstituted membranes that were purified by floatation in sucrose gradients tended to be lower than the protection induced by the non-purified reconstituted membranes, but this reduction was not statistically significant.

\section{Trypsin treatment of solubilized membrane proteins}

To determine whether the immunoprotective activity observed with reconstituted membranes could be attributed to (glyco)proteins, solubilized membranes were treated with the proteolytic enzyme trypsin. Reconstituted membranes were formed by removal of the detergent and were purified by floatation in a discontinuous sucrose gradient to remove trypsin. No protection against a challenge with SL2 cells was observed after immunization of mice with the trypsindigested preparations; immunization of mice with nontrypsinized purified reconstituted membranes elicited significant protection $(P<0.05)$ (Table 2$)$.

\section{Reconstituted membranes containing additional exogenous phospholipid}

The effect of the addition of exogenous PL on the characteristics and the immunogenicity of reconstituted membranes was studied. To solubilized SL2 membranes, different amounts of PtdCho/PtdGro (9:1), also solubilized in OG, were added. After dialysis, the reconstituted membranes were purified by floatation in sucrose gradients. The percentage protein recovered in the upper three fractions increased significantly $(P<0.01)$ from $31 \%$ (no exogenous $\mathrm{PL}$ ) to $48 \%$ (addition of $30 \mu \mathrm{mol}$ exogenous $\mathrm{PL} / \mathrm{ml}$ solubilized membranes) (Fig. 6).

The distribution of proteins and PL of reconstituted membranes and of reconstituted membranes with $30 \mu \mathrm{mol}$ $\mathrm{PL} / \mathrm{ml}$ solubilized membranes in a continuous sucrose gradient is shown in Fig. 7. For each sucrose density gradient profile, the protein-to-PL ratio increased with increasing density. Maximal concentrations of PL were found in fractions 3-5 (density 1.06-1.13) for reconstituted membranes and in fraction 1 (density 1.02) for reconstituted
A: without exogenous phospholipid

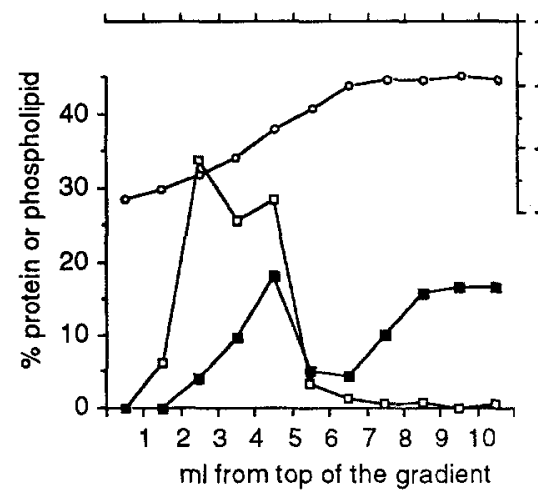

$\mathrm{ml}$ from top of the gradient
B: with exogenous phospholipid

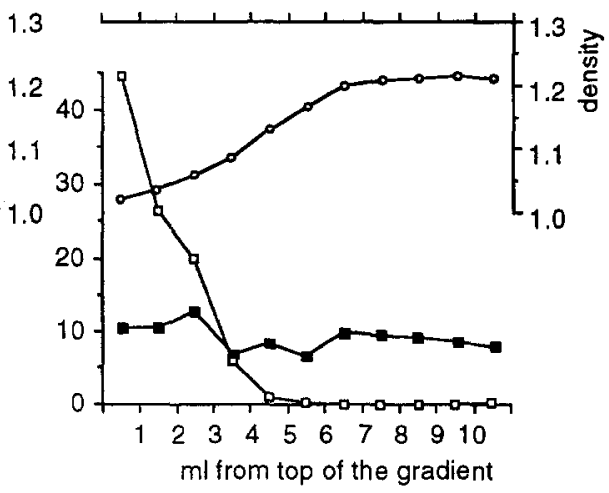

Fig. 7 A, B. Floatation of reconstituted membranes and reconstituted membranes with exogenous phospholipid in a continuous sucrose gradient. Reconstituted membranes (A) and reconstituted membranes prepared with $30 \mu \mathrm{mol}$ exogenous phospholipid/ml solubilized membranes (B) were applied to the bottom of a continuous sucrose gradient. After centrifugation for $16 \mathrm{~h}, 1$-ml fractions were collected from the top of the gradient and analysed for protein (ש) and phospholipid ( $\square$ ) content. O, Density. Typical experiment out of two performed is shown 
Table 3. Protective tumour immunity induced by immunization with purified reconstituted membranes containing additional exogenous phospholipids

\begin{tabular}{|c|c|c|c|}
\hline \multicolumn{2}{|c|}{ Purified reconstituted membranes ${ }^{a}$} & \multicolumn{2}{|c|}{ Surviving mice at dayb } \\
\hline $\begin{array}{l}\text { Phospholipid added } \\
\text { ( } \mu \text { mol/ml solubilized } \\
\text { membranes) }\end{array}$ & $\begin{array}{l}\text { Injected } \\
\text { phospholipid } \\
\text { dose }(\mu \mathrm{mol})\end{array}$ & 40 & 90 \\
\hline 0 & 0.1 & $5 / 13(38)$ & $4 / 13(31)$ \\
\hline 3 & 0.5 & $4 / 10(40)$ & $3 / 10(30)$ \\
\hline 10 & 1.3 & $3 / 10(30)$ & $2 / 10(20)$ \\
\hline 30 & 2.5 & $2 / 15(13)$ & $2 / 15(13)$ \\
\hline
\end{tabular}

a Reconstituted membranes were prepared with the indicated amount of phospholipid (phosphatidylcholine/phosphatidylglycerol ratio of 9:1) and centrifuged in a discontinuous sucrose gradient. The upper three fractions containing the purified reconstituted membranes were pooled, dialysed and concentrated. Mice were immunized three times s.c. (10day intervals) with $40 \mu \mathrm{g}$ protein and challenged i. p. with $3 \times 10^{2}$ SL2 cells 10 days after the last immunization

b Number of animals alive at the day indicated/total number of animals (\% within parentheses). Naive control mice injected with the same tumour challenge died within 25 days

membranes with exogenous PL. Thus, addition of exogenous PL resulted in an increased number of vesicles with a relatively low protein content. However, the density profiles also indicate that the purified reconstituted membranes used for the immunization studies contained vesicles with a variety of protein-to-PL ratios. This may also apply to the unpurified reconstituted membranes, although the osmotic pressure produced by the high sucrose concentrations may affect the vesicle structure during gradient centrifugation. A slight increase in mean particle size and polydispersity index of the reconstituted membranes was generally observed after purification by floatation in the discontinuous sucrose gradients (Table 1).

Mice were immunized with purified reconstituted membranes and purified reconstituted membranes with 3,10 or $30 \mu \mathrm{mol}$ exogenous PL. No significant differences were found in the potential of these preparations to induce protective tumour immunity (Table 3 ).

\section{Discussion}

TAA were extracted from crude membranes of SL2 cells and subsequently associated with additional extracted PL to form a membrane-like, well-defined presentation vehicle. Substantial attention was paid to a proper physicochemical characterization of these reconstituted membranes. Although this method has often been used for reconstitution of major histocompatibility antigens and viral proteins [6], it has not been explored before for tumour vaccine preparation. The reconstituted membranes were more immunogenic in terms of induction of protection than crude membranes when compared on the basis of protein dose (Fig. 5).

For the extraction of proteins and lipids from SL2 cells OG was employed. This detergent solubilizes selectively about $40 \%$ of the SL2 membrane proteins, including the supposed TAA. On SDS-PAGE, the protein bands of the reconstituted membrane samples were also found in the crude membrane samples. Upon storage of membranes and reconstituted membranes at $-20^{\circ} \mathrm{C}$, no alterations in the protein pattern were seen over 3 months. This indicates that major proteolytic degradation of proteins does not occur under these circumstances. Proteolytic degradation of proteins during extraction and upon storage has been reported for $3 \mathrm{M} \mathrm{KCl}$ extracts [31]. As freezing/thawing of reconstituted membranes can induce changes in vesicle structure, they were not stored in frozen condition, but freshly prepared for each immunization.

Reconstitution of the membrane proteins with the PL from the membranes cosolubilized has the advantage that - besides the simplicity of the method - the mixture of lipids is more likely to contain any essential lipids or to approximate closely any particular lipid requirement than would a defined mixture of highly purified lipids [8]. This may result in higher reconstitution efficiencies. For instance, Hudson et al. [20] reported a poor reconstitution of Plasmodium knowlesi merezoite antigen into liposomes containing a synthetic PL, but the antigen was reconstituted effectively by liposomes containing the same PL composition that was previously reported to be present in P. knowlesi.

It was reported earlier [3] that about $50 \%$ of the proteins present in the reconstituted membrane preparation could be pelleted by high-speed centrifugation. Centrifugation caused aggregation of the vesicles. It was not possible to resuspend the pelleted vesicles to assess their immunogenic properties. Therefore, in the present study, reconstituted membranes were purified by floatation in a discontinuous sucrose gradient, and divided into two fractions for immunoprotection studies. About $30 \%$ of the proteins and $90 \%$ of the PL applied to the gradient were recovered in the upper fraction, containing proteins associated with reconstituted membranes (fractions $1-3$ in Fig. 1 A). Protection studies indicated that this fraction contained a considerable amount of immunogenic material. However, immunization of mice with the remaining (bottom) fraction (fractions 6-11 in Fig. 1A) elicited protection as well. Analysis of this fraction revealed that about $28 \%$ of the proteins present in this fraction could be pelleted by highspeed centrifugation. Immunization of mice with the remaining fraction after removal of particulate material did not affect tumour growth. Therefore, it can be concluded for the (unpurified) reconstituted membranes that particulate material is essential for the induction of immune responses. A major part of the immunogenic activity of this particulate material can be attributed to proteins associated with the reconstituted membranes. Soluble material is not very immunogenic by itself, although such material present in the reconstituted membranes may contribute to the immunological response. An additional effect of free protein on the immune response induced by liposome-associated protein has been reported by Ho et al. [18] for herpes simplex virus glycoprotein $\mathrm{D}$.

Purified reconstituted membranes presented in a solubilized form were not immunogenic (Fig. 4). As a high detergent-to-protein ratio was used ( $150: 1$ by weight) this preparation contained probably protein-detergent complexes 
and lipid-detergent mixed micelles [17] instead of liposomal structures. These results resemble the low immunogenicity of protein-detergent complexes of spike glycoproteins of virus envelopes $[1,28]$. This has been ascribed to the monomeric form of the protein-detergent complex; presentation of spike glycoproteins in multimeric forms (viruses, liposomes, protein micelles) was far more effective. Supersaxo et al. [37] showed that following s. c. injection of drug/PtdCho/bile salt mixed micelles, the micelles were converted in vivo into liposomes, probably because of a combined effect of dilution and dialysis. Drugs with a high lipophilicity were associated with the newly formed liposomes. This mechanism is not likely to occur after injection of the solubilized reconstituted membranes, as a high detergent-to-protein ratio and very low doses of protein and PL were employed. Injection of the OG-solubilized membrane proteins or only OG caused severe inflammation at the injection site, leading to ulceration. These results show that detergent removal is required to obtain specific stimulation of immune responses by SL2 membrane TAA and to avoid the toxicity associated with the detergent.

The experiments concerning the addition of exogenous PL to the solubilized membranes were performed for two reasons. First, addition of exogenous PL might increase the reconstitution efficiency as determined by sucrose gradient centrifugation. Studies on the reconstitution of purified hydrophobic proteins (e. g. rhodopsin, major histocompatibility antigens, viral antigens) into liposomes have shown that much lower protein-to-PL ratios are required for reconstitution than are present in biological membranes [13]. The exact reason is not understood. The reconstitution efficiency of solubilized SL2 crude membrane proteins, with an initial protein-to-PL ratio of $0.8 \mathrm{mg} / \mu \mathrm{mol}$, increased slightly on the addition of exogenous PtdCho/PtdGro to a protein-to-PL ratio of $0.03 \mathrm{mg} / \mu \mathrm{mol}$ (Fig. 6).

Second, the protein-to-PL ratio may affect the immunogenicity of liposome-presented antigens, as described for the humoral response against protein antigens [10, 38]. Liposomal incorporation of crude or semi-purified TAA extracts can augment specific immune reactions against TAA $[16,25,30,33,34]$. In these studies a variety of tumour models, extraction techniques, and liposome types has been employed. They have in common that the liposomes contained much lower protein-to-PL ratios (generally between 0.01 and $0.04 \mathrm{mg} / \mu \mathrm{mol}$ ) than the reconstituted membranes $(0.3 \mathrm{mg} / \mu \mathrm{mol})$ used in the study presented here. Consequently, higher PL doses are required to inject an equal dose of protein. It is possible that liposomes with a low protein-to-PL ratio may induce enhanced immune responses, either by a low concentration of antigen in individual vesicles or by the mass of injected liposomal lipid. Reconstitution of SL2 membrane proteins with additional PL resulted only partially in liposomes with a lower protein content. Floatation of the liposomes in continuous sucrose gradients revealed that protein-rich liposomes exist next to protein-poor liposomes (Fig. 7). This phenomenon is not exclusively for reconstitution of crude mixtures of membrane proteins. Several investigators have reported a non-random distribution of purified proteins in liposome dispersions [e.g., 12, 21]. In the publications mentioned above on TAA extracts in liposomes, the homogeneity of the dispersions was not described. No major differences in the immune responses elicited by reconstituted membranes and reconstituted membranes with additional PL were observed in the present study.

Almost all the immunoprotective TAA that have been identified up to now are (glyco)proteins [35]. (Glyco)proteins were the essential TAA in the reconstituted membranes as well, as digestion of SL2 membrane proteins with trypsin destroyed the ability to induce protection. This is consistent with the earlier observation that mice immunized with reconstituted membranes mainly consisting of lipids or with semi-purified glycolipids of SL2 cells incorporated into liposomes could not reject the tumour [3].

In conclusion, membrane presentation of TAA on reconstituted membranes obtained by solubilization of crude SL2 membranes with OG, and subsequent removal of the detergent, is more effective in terms of the induction of immunoprotection than presentation of TAA on crude membranes. Detergent removal is critical for the induction of protective immune responses. Although the dispersion showed a degree of heterogeneity, the procedures described here may be a good starting-point to obtain strongly immunogenic TAA following a standard preparation procedure as: (a) peripheral and transmembrane proteins and glycolipids are obtained with minimal contamination with intracellular proteins, (b) OG is a "gentle", non-ionic detergent, causing minimal protein denaturation; OG can be easily removed by dialysis, (c) proteins solubilized by $O G$ can be further purified in the "solubilized state", (d) antigens are presented on a membrane-like structure, which also allows evaluation of membrane factors and of accessory molecules necessary for the induction of immune responses, (e) injection of toxic detergent is prevented, and (f) amphipathic adjuvants may be co-incorporated into the reconstituted membranes to enhance the induced immune response. They may provide an alternative for whole-cell vaccines in immunotherapeutic trials and for studying the interaction of TAA with cells of the immune system in order to manipulate immune responses.

Acknowledgements. We would like to thank Dr. J. Röding (Nattermann Phospholipid GmbH, Cologne, Germany) and his assistants for providing the electron micrographs.

\section{References}

1. Balcarova J, Helenius A, Simons K (1981) Antibody response to spike protein vaccines prepared from Semlike Forest virus. J Gen Virol 53: 85

2. Balch CM, Riley LB, Bae YJ, Salmeron MA, Platsoucas CD, Von Eschenbach A, Itoh K (1990) Patterns of human tumor-infiltrating lymphocytes in 120 human cancers. Arch Surg 125: 200

3. Bergers JJ, Den Otter W, De Groot JW, De Blois AW, Dullens HFJ, Steerenberg PA, Crommelin DJA (1992) Reconstituted membranes of tumour cells (proteoliposomes) induce specific protection to murine lymphoma cells. Cancer Immunol Immunother 34: 233

4. Bergers JJ, Storm G, Den Otter W (1993) Liposomes as vehicles for the presentation of tumor-associated antigens. In: Liposome technology, vol II, 2nd edn. CRC Press, Boca Raton, Fla. p 141 
5. Bligh EG, Dyer WJ (1959) A rapid method of total lipid extraction and purification. Can J Biochem Physiol 37: 911

6. Burakoff SJ, Mescher MF (1982) Reconstituted membranes and liposomes in the study of lymphocyte interactions. Cell Surface Rev 8: 173

7. Bystryn J-C (1990) Tumor vaccines. Cancer Metastasis Rev 9: 81

8. Cerione RA, Ross EM (1991) Reconstitution of receptors and G proteins in phospholipid vesicles. Methods Enzymol 195: 329

9. Cornet B, Vandenbranden M, Cogniaux J, Giurgea L, Dekegel D, Ruysschaert J-M (1990) Virosomes reconstituted from human immunodeficiency virus proteins and lipids. Biochem Biophys Res Commun 167: 222

10. Davis D, Gregoriadis G (1987) Liposomes as adjuvants with immunopurified tetanus toxoid: influence of liposomal characteristics. Immunology 61: 229

11. Dullens HFJ, Hilgers J, Spit BJ, De Heer E, De Weger RA, Van Basten CDH, Den Otter W (1982) Staging, growth properties and metastatic behaviour of a transplantable murine T-cell lymphoma. Int J Tissue React 4: 15

12. Engelhard VH, Guild BC, Helenius A, Terhorst C, Strominger JL (1978) Reconstitution of purified detergent-soluble HLA-A and HLA-B antigens into phospholipid vesicles. Proc Natl Acad Sci USA 75: 3230

13. Eytan GD (1982) Use of liposomes for reconstitution of biological functions. Biochim Biophys Acta 694: 185

14. Fiske $\mathrm{CH}$, Subbarow $\mathrm{Y}(1925)$ The colorimetric determination of phosphorus. J Biol Chem 66: 375

15. Gregoriadis G (1990) Immunological adjuvants: a role for liposomes. Immunol Today 11: 89

16. Hedlund G, Jansson B, Brodin T, Sjögren HO (1988) In vivo use of liposome-incorporated membrane antigens. In: Liposomes as drug carriers; recent trends and progress. Wiley, New York, $\mathrm{p} 167$

17. Hjelmeland LM, Chrambach A (1984) Solubilization of functional proteins. Methods Enzymol 104: 305

18. Ho RJY, Burke RL, Merigan TC (1990) Physical and biological characterization of antigen presenting liposome formulations: relative efficacy for the treatment of recurrent genital HSV-2 in guinea pigs. Antiviral Res 13: 187

19. Hoover HC, Hanna MG (1991) Immunotherapy by active specific immunization: clinical applications. In: Biological therapy of cancer. Lippincott, Philadelphia, p 670

20. Hudson DE, Miller LH, Richards RL, David PH, Alving CR, Gitler C (1983) The malaria merozoite surface: A 140,000 M. W. protein antigenically unrelated to other surface components on Plasmodium knowlesi merozoites. J Immunol 130: 2886

21. Jackson ML, Litman BJ (1982) Rhodopsin-phospholipid reconstitution by dialysis removal of octylglucoside. Biochemistry 21: 5601
22. Jiskoot W, Van Hertrooij JJCC, Klein Gebbinck JWTM, Van der Velden-de Groot T, Crommelin DJA, Beuvery EC (1989) Two-step purification of a murine monoclonal antibody intended for therapeutic application in man. Optimisation of purification conditions and scaling up. J Immunol Methods 124: 143

23. Kersten GFA, Beuvery EC, Crommelin DJA (1989) Antigen presentation and adjuvants. In: Topics in pharmaceutical sciences. Amsterdam Medical Press, Noordwijk, The Netherlands, p 313

24. Laemmli UK (1970) Cleavage of structural proteins during the assembly of the head of bacteriophage T4. Nature 227: 680

25. LeGrue SJ (1984) Carrier and adjuvant properties of liposome-borne tumor-specific antigens. Cancer Immunol Immunother 17: 135

26. LeGrue SJ (1985) Noncytolytic extraction of cell surface antigens using butanol. Cancer Metastasis Rev 4: 209

27. Marx JL (1989) Cancer vaccines show promise at last. Science 245: 813

28. Morein B, Helenius A, Simons K, Pettersson R, Kääriäinen L, Schirrmacher V (1978) Effective sububit vaccines against an enveloped animal virus. Nature 276: 715

29. Old LJ (1981) Cancer immunology: the search for specificity. Cancer Res 41: 361

30. Phillips NC, Loutfi A, Kareem M, Shibata H, Baines M (1989) Experimental and clinical evaluation of liposome-tumor antigen immunotherapy. In: Liposomes in the therapy of infectious diseases and cancer. Liss, New York, p 15

31. Prat M, Tarone G, Comoglio PM (1975) Antigenic and immunogenic properties of membrane proteins solubilized by sodium desoxycholate, papain digestion or high ionic strength. Immunochemistry 12:9

32. Prehn RT, Main JM (1957) Immunity to methylcholanthrene-induced sarcomas. JNCI 18: 769

33. Schroit AJ, Key ME (1983) Induction of syngeneic tumour-specific immunity by liposomes reconstituted with $\mathrm{L}_{2} \mathrm{C}$ tumour-cell antigens. Immunology 49: 431

34. Shibata R, Noguchi T, Sato T, Akiyoshi K, Sunamoto J, Shiku H, Nakayama E (1991) Induction of in vitro and in vivo anti-tumor responses by sensitization of mice with liposomes containing a crude butanol extract of leukemia cells and transferred intermembranously with cell-surface proteins. Int J Cancer 48: 434

35. Srivastava PK (1991) Protein tumor antigens. Curr Opin Immunol 3: 654

36. Stevenson FK (1992) Update on tumor vaccines. Int J Clin Lab Res 22: 84

37. Supersaxo A, Hein WR, Steffen H (1991) Mixed micelles as a proliposomal lymphotropic drug carrier. Pharmaceutical Res 8: 1286

38. Thérien H-M, Shahum E, Fortin A (1991) Liposome adjuvanticity: influence of dose and protein: lipid ratio on the humoral response to encapsulated and surface-linked antigen. Cell Immunol 136: 402

39. Wessel D, Flügge UI (1984) A method for the quantitative recovery of protein in dilute solution in the presence of detergents and lipids. Anal Biochem 138: 141 